\title{
COGNITIVE FACILITIES MANAGEMENT: DEFINITION AND ARCHITECTURE
}

\author{
$J X u^{*}, W . L u$ and L.H. $L i$ \\ Department of Real Estate and Construction, The University of Hong Kong, Hong Kong, China \\ * Corresponding author
}

\begin{abstract}
Prevailing facility management (FM) discourses increasingly recognise the importance of facilities in improving the quality of our life and the productivity of our core business. Nevertheless, no matter how intelligent or smart they are advocated, facilities are, by and large, treated as 'aliens' to us, human beings. This problem can be reduced by enhancing cognition ability of FM. Cognition is the process of acquiring knowledge and understanding through perceptions, action, and learning. Cognitive facilities management can percept through the cognitive Internet of Things, learns in the manner of human cognition with the power of cognitive computing, and acts actively, adaptively, and efficiently via robotics or automated actuators to improve the quality of people's life and productivity of core business. The architecture of Cognitive FM proposed in this study consists of eight layers, namely, object layer, sensing layer, data layer, communication layer, computing layer, application layer, actuation layer, and evaluation layer, sequentially from lower to upper. Through these layers, two information cycles take shape. The perception-action cycle between object and actuation layer enables targeted response, whilst evaluation-adapting cycle between evaluation and computing layer facilitates adaptive learning. With the architecture and technologies behind, the CFMS attains three smart properties, i.e., awareness, communicativeness, and autonomy. The contributions of this Cognitive FM architecture are twofold: (1) promotes a potential approach towards advanced intelligence in FM; and (2) provides a detailed architecture to develop practical, smart FM applications.
\end{abstract}

\section{Introduction}

A facility is an asset that is built, installed or established to serve social and economic activities (Kaplan and Norton 2004). In this paper, facilities include different types of physical infrastructures (e.g., bridges, roads) and buildings (e.g., hospitals, shopping malls, residential buildings). The management of facilities, i.e., facility management (FM), is "a profession that encompasses multiple disciplines to ensure functionality, comfort, safety, and efficiency of the built environment by integrating people, place, process, and technology" (IFMA, 2017). Similarly, ISO (2017) defined FM as the organisational function that integrates people, place, and process within the built environment with the purpose of improving the quality of life of people and the productivity of the core business. It is crucial to facilitate resources use, enhance organisational effectiveness, and improve our living and working environment for social and economic activities (Chotipanich, 2004; Rondeau et al., 2012; Alexander, 2013). Compared with other phases of a building life cycle, FM can last for a relatively long period, thirty to fifty years if not longer (Atkin and Brooks, 2015). Therefore, the efficiency and effectiveness of FM can significantly affect the operation cost (Teicholz, 2013), energy consumption (Bilgen, 2014), and even the global climate (Nielsen et al., 2016).

As a famous saying of Churchill goes: "We shape our buildings, thereafter they shape us", buildings and human beings are closely intermingled with each other. Over the last three decades, smart facility and its management captured the cornerstone of FM development, especially with the lasting and fierce discussion of smart building and intelligent building.
Nevertheless, current FM practices, ranging from safety surveillance to indoor environment management, are largely backward (Pärn et al., 2017), characterised by low level of automation and intelligence (Wu et al., 2014). They often perform passively in an isolated way (Lau et al., 2013). For example, it is common to see different FM systems, e.g., communication automation system (CAS), building automation system (BAS), firefighting alarm system (FAS), and security automation system (SAS), do not share relevant information. Moreover, the decision-making and execution of FM heavily rely on manual work, which can be error-prone due to the computation capacity and bounded rationality of human beings (Niu et al., 2016; Cheng et al., 2017). More seriously, current FM generally pays scant attention to the differentiated requirements of occupants. Therefore, it cannot provide satisfactory services to people and organisations it serves (Wang et al., 2018).

Motivated by the cognitive system, FM needs to be developed and upgraded in order to satisfy the efficient and effective delivery of services and free people from redundant FM practices. The cognitive system is a system that can be aware of its behaviour and capabilities and modify its behaviour by experience in the face of unexpected natural and social circumstances (Woods and Hollnagel, 2005; Sheth, 2016). A human brain is a typical cognitive system that can process fuzzy data analysis and make proactive decisions in complex situations. If an FM system is to be cognitive, advanced technologies must be adopted to access data, process data, and make decisions. Although some recent research efforts have explored the use of advanced information technologies to support FM, none of them has touched upon equipping 
facilities with cognitive ability to let them percept their environment, capacity, and behaviour; communicate amongst the whole system; learning patterns and relationships; and actively respond to various scenarios.

This study thus aims to take the very first step to illustrate a new, comprehensive FM mode- cognitive FM - that fully integrated cognitive system into the specific FM practices, and answer the following research questions: (1) what is cognitive FM? (2) what are the essential elements of cognitive FM? and (3) what is the architecture of cognitive FM? The remainder of this paper is organised as follows: Section 2 reports related work on emerging technologies for FM and cognitive system. Section 3 discusses the definition, key elements and enabling technologies of cognitive FM. The proposed architecture of cognitive FM is illustrated in Section 4. Conclusions are drawn in Sections 5, respectively.

\section{Related work}

\subsection{Advanced technologies for facility management}

Previous studies have used many different advanced technologies to support FM practices. These efforts can be briefly summarised into three categories as follows.

Using data sensing technologies such as laser scanning and photogrammetry to reconstruct digital models of facilities (Wong et al., 2018). A hand of studies focuses on constructing as-built/as-is digital models of facilities with 3D point cloud data collected by laser scanners or images taken by cameras (Xu et al., 2018). With their efforts, more and more semiautomatic or even automatic reconstruction methods to create as-built/as-is models are developed (Tang et al., 2010; Yang et al., 2013). A complete as-built/as-is building information model (BIM) of facilities can support applications such as asset management (Leite and Akinci, 2011; Cavka et al., 2017), indoor navigation (Isikdag et al., 2013), and emergency management (Li et al., 2014; Cheng et al., 2017). However, the majority of previous studies only reconstructed the exterior surface and structural elements of the buildings, but only a few focused on the perception of what that information means to FM.

Using advanced communication technologies and actuation devices to control the HVAC systems and lighting systems according to the occupants' behaviours (Jazizadeh et al., 2014). Studies of this kind monitor HVAC system based on bespoke thermal comfort of occupants (Kim et al., 2018) and the lighting system according to their luminous comfort (Xue et al., 2014). For example, Ghahramani et al. (2014) coupled personalised thermal comfort preferences with energy consumption patterns to improve HVAC system operations. Yang and Becerik-Gerber (2014) utilised a long-term occupancy profile to schedule HVAC system. However, it is inadequate and incomplete to use binary occupancy status (i.e., occupied/unoccupied) (Yang and Becerik-Gerber, 2014) and static range of thermal comfort preference by scale survey (Ghahramani et al., 2014) to measure the performance of existing systems. Consequently, it is neither reliable nor efficient to set up new HVAC operation rules based on the shallow data to improve energy efficiency (Xu et al., 2017). Moreover, these pre-set controls are not flexible and adaptive to the complex and dynamic environment of the facilities.

Using information technologies to develop systems, platforms, and applications to visualise information of FM ( $\mathrm{Lu}$ et al., 2018). Apart from traditional CMMS (computerised maintenance management system) and CAFM (computeraided facility management) systems, commercial ones like the Onuma system, Archibus, FM:systems, Bentley Facilities, BIM 360, Vintocon ArchiFM, IBM Tririga, and EcoDomus were developed (Wong et al., 2018). These systems integrate different types of data and information from different sources and provide solutions for space management, workplace management, asset management, real estate management, facility maintenance, for example. In academia, $\mathrm{Hu}$ et al. (2018) developed a cross-platform operation and maintenance management system using the MEP-related information in the as-built model to effectively response to MEP-related emergencies. Nevertheless, most of these systems fail to comprise the various requirements from all aspects of FM (Wong et al., 2018), never to say learn patterns and relationships from the information.

\subsection{Cognitive system}

A cognitive system can percept, reason, use represented knowledge, learn from its experience, accumulate knowledge, explain itself, accept directions, be aware of its behaviour and capabilities as well as take action in a way that loosely mimics the process of cognition in the human mind (Sheth, 2016). Currently, the cognitive system is still at its infancy, where most research focuses on the discussion of its understanding and design. IBM with the so-called "Cognitive Building" aiming at better energy use management, space utilisation, operating cost reduction, real estate planning and management simplification applying the cognitive system to AEC/FM domain. However, its focus is more on commercial buildings, and scant attention was paid to the learning ability of buildings.

Cognitive computing (CC) enables cognitive system the fuzzy computing ability. It is an interdisciplinary product that combines cognitive science and computer science to mimic human thinking process in a computerised model. CC pursues approaching the low power, small volume, mind-like function, and real-time performance of the human brain (Preissl et al., 2012). It combines data mining with predictive analysis to forecast future events with estimated strength (Marchevsky et al., 2017). It can extract concepts, emotions, objects, keywords, and relationships from unstructured data, and use enhanced machine learning algorithms to process analytics, make predictions and hypothesis in a scalable way (Dessì et al., 2018). Natural language processing, machine reasoning, speech recognition, computer vision, human-computer interface, and knowledge discovery all belong to the subfield of CC (Capuano and Toti, 2018).

Cognitive Internet of Things (CIoT) provides a cognitive system with fuel to percept itself and its environment. As a particular sub-area of IoT, CIoT aims to incorporate cognitive ability into traditional IoT (Feng et al., 2017). According to $\mathrm{Wu}$ 
et al. (2014), CIoT is a new network paradigm, where both physical and virtual objects are interconnected and behave as agents, with minimum human intervention. Agents in CIoT interact with each other and learn from the physical environment and social networks; keep the learned semantic and knowledge in various databases; and adapt themselves to changes or uncertainties via resource-efficient decisionmaking mechanisms empowering smart resource allocation, automatic network operation, and intelligent service provisioning. Attempts were made to apply CIoT to concrete transportation and dispatching system (Zhang et al., 2012), smart city (Somov et al., 2013), smart home (Feng et al., 2017), and amateur drone surveillance system (Ding et al., 2018).

After revisiting research on emerging technologies for FM and cognitive systems, the research gaps can be identified. Firstly, though many efforts are made to the development from conventional FM to advanced FM, most of them attend to the physical (e.g., HVAC systems) and cyber spaces (e.g., 3D digital model). Social activities and people's requirements still have not received enough attention. However, according to recent popular concept CPSS (cyber-physical-social system), human beings (the service target and focal point of FM decision-making) and social activities (the functional exemplification of facilities) should never be neglected by any FM systems. Secondly, no research was found to try to integrate the cognitive system into FM. Therefore, little research is found to address the problem of how to give cognition to facilities. This paper aims to fill the gaps by applying CIoT and cognitive computing to FM, putting forward the concept of cognitive FM, and presenting a potential architecture.

\section{Cognitive FM}

\subsection{Definition}

Based on the review of advanced technologies for FM and cognitive systems, Cognitive FM is defined as:

The active intelligent management of a facility, which can perceive through cognitive systems, learn in the manner of human cognition with the power of cognitive computing, and act actively, adaptively, and efficiently via automated actuators, to improve the quality of people's life and productivity of core business.

Cognitive FM will give old FM a new life in the era of intelligence. Perception, learning, and action are the three key elements of cognitive FM. Perception is the process that transforms raw input into the system's internal representation for carrying out cognitive tasks (Kotseruba and Tsotsos, 2018). In cognitive FM, Cognitive IoT percepts the environment indicators, the operation status of devices and utilities, user behaviour, location and identities, and facility information from raw data captured by sensor networks, cameras, laser scanners, Auto-IDs, GPS, and smart devices. Learning is the capability of improving a system's performance over time (Kotseruba and Tsotsos, 2018). CC fuels cognitive FM with the ability to learn accurately, efficiently, and flexibly to adjust the facility to different requirements and preference of users, to monitor the operations of multiple systems, with the consideration of energy efficiency, service quality, and business productivity. The action is what to do next and how to deal with inputs from a system itself and its environments. It may refer to goal, task, command or execution. In Cognitive FM, action can be the turning up/down of the thermostat temperature, sending fire alerts, or scheduling maintenance work. The three key elements, i.e., perception, learning, and action, have a direct or indirect influence on each other with the changing environment and requirements from users.

\subsection{Key elements and their enabling technology}

In the cognitive system, there are several essential elements, including receptors, motor control, perception, action, memory, attention, feedback, and learning (Norman, 1980). This paper selects the three representative and primary elements, i.e., perception, action, and learning, as key elements of Cognitive FM. Because receptor is a premise part of perception, motor control is an extended part of the action, while memory, attention, and feedback are indispensable for perception, action, and learning.

Figure 1 shows the details and relationship of the three key elements, i.e., perception, action, and learning, of Cognitive FM. As Gibson (2015) argued: "We must perceive in order to move, but we must also move in order to perceive", perception and action are intricately connected. Perception provides the requisite information for selecting and guiding actions adaptively (Franchak et al., 2010). Action, in turn, has concurrent effects on perception (Hecht et al., 2001). They form a perception-action cycle through connections between sensory and motor structures (Fuster, 2003). The cognitive interactions of a system (be it animate or artificial) with the surrounding world are governed by the perception-action cycle. This interactive cycle is the basic principle that characterises the dynamic adaptation of the organism to its environment (Fuster, 2003). It embodies a feedback-based decision-making mechanism, which can identify intelligent choice in complex and uncertain environments (Haykin, 2012). Hidden behind the perception-action cycle to make a system cognitive is learning. Learning helps the system improve its performance on future actions after making perceptions about the internal and external environment (Russell and Norvig, 2016). Learning from perceptions empowers reasoning and knowledge discovery about the changing of the internal and external environment. Learning from prior knowledge and feedbacks facilitate the system to make adaptations to uncertainties, to make decisions that maximise the benefits and minimise the risks. Learn from a collection of perceptionaction cycles enables the system to predict actions and select the most appropriate one for a new perception. In FM, perception, learning, and action form a stable triangle that helps a facility to accurately, effectively, and actively adapt to the dynamic environments and various requirements of users. 
Figure 1 The key elements of a Cognitive FM

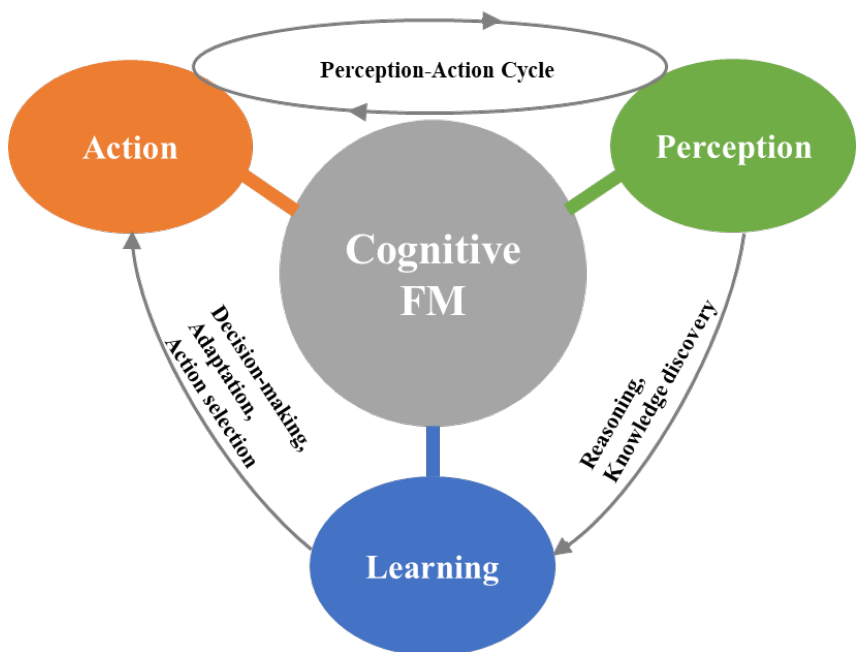

3.2.1 Perception: Perception is a lot more than sensing or capturing an incoming stimulus (Ratey, 2001). It is an active process, which involves the recognition and interpretation of stimuli that register on senses that are made on the environment (Rookes and Willson, 2005). It requires a form of expectation, of knowing what is about to confront us and preparing for it (Ratey, 2001). In a word, perception provides the system with information about the world it inhabits by interpreting the response of sensors (Russell and Norvig, 2016). According to theories of perception, there are some matching process between sensory information and stored representation for final identification to take place. The stored representation is knowledge predefined or learned from experience. For example, a temperature sensor reports a number 18, perception is the process to interpret it together with other factors to meaningful information that the current indoor temperature of the room is $18^{\circ} \mathrm{C}$, which is a bit low for people inside.

Perception can be empowered by Cognitive IoT (CIoT), which supports the cooperation and interaction between IoT and human. Wired or wireless sensors, cameras, LiDAR, Auto-IDs, and GPS, smart devices and they connected utilities are the "things" that can sense the status and environments of facilities. Wireless sensor networks (WSNs) in buildings, utilities, transportation systems can enable the in-time collection of sensory data (Huang and Mao, 2017). Videos or images captured by cameras are broadly used in gathering movement and behaviour information for health, safety, and security. They are also pervasive sources of spatial and geometric data for reconstructing digital models of in-use facilities. LiDAR captured 3D point cloud data is another source of as-built facility digital model. Auto-IDs such as 2D barcode and RFID, as well as GPS, make real-time localisation, tracking, and navigation at fingertips. Being capable of collecting different types of data, such as the ambient environment, user behaviour patterns, and so forth, smart devices become a growing information source. Cognitive computing sponsored CIoT to have the ability to percept the various information of the facility, users and the environment. Once widely deployed, CIoT will turn accurate and complete perceptions of dynamic complex conditions simple.
3.2.2 Learning: Learning is the accumulation of information in memories, the acquisition of access routes for retrieving it, and the discovery of knowledge based on it (Russell and Norvig, 2016). In a cognitive system, learning helps to recognise objects, categorisations, relationships, events, procedures for executing actions, new actions and action sequences to accomplish new tasks (Franklin et al., 2014). It provides an enormous source for variation, adaptation, and evolution in system action. For example, analysing historical data of the indoor temperature and the control of air conditioner of a room, learning helps the system finds out what is the preference of indoor temperature for the people in that room.

Learning has two types, i.e., selectionist (learning by the reinforcement of existing representations) and instructionalist (learning by the creation of new representations) (Franklin et al., 2014). Both can be achieved through four approaches, including observation, experience, feedback, and reinforcement. Watching other's performance and imitating, concluding experiences and recognising patterns, reflecting feedbacks and discovering knowledge, and reinforcing by punishment or rewards. In real cases, learning is a multimodal process that integrates multi approaches and mechanisms. Multimodal integration learning combines multiple sources of sensory information such as vision, sound, movement to help a cognitive system achieve enhanced perceptual clarity and reduced ambiguity regarding its environment (Noda et al., 2014)

The engine of learning in the cognitive system lies in the new wave of cognitive computing (CC) (Coccoli et al., 2016). CC implements computational intelligence by autonomous inferences and perceptions mimicking the mechanisms of the brain (CC). With the fundamental technologies in CC, such as machine learning (ML), computer vision (CV), natural language processing (NLP), sentiment analysis, and neural networks, learning of a Cognitive FM will be energised with the fuel of gigantic amount of data and information from perceptions, actions, and perception-action cycle. This whole learning process follows the way people learn.

3.2.3 Action: Action in human cognition is instantiated in the brain as a motor planning and representation (ImmordinoYang, 2008). However, in cognitive FM, an action is more than mental, it may include decision making, alert sending, function recommending, statistics analysing and visualising, model reconstructing, service-provisioning, device actuating, and so forth. For example, an action is, after learning the temperature preference of the people in the room, adjusting the air conditioner to keep the room at the preferred temperature. Merely being in action can facilitate the perception of affordances because actions provide information about the dynamic capabilities of the facility (Franchak et al., 2010).

In cognitive FM, action can be executed in three ways: passive, semi-active and active. Passive action aims to assist people in making decisions with an excellent action plan based on the real-time situation it percepts, such as issuing an alert in seek for the response from facility managers or workers. Semiactive action is an action that cooperated by human and machine via the human-machine interactions. An instance can 
be: a facility manager wants to know the energy consumption of a specific room, he can ask Cognitive FM, and the system will give him the results. Active action can execute the optimum action plan by the facility itself, without including humans in the loop. For example, controlling the lights of a room based on occupancy, natural light condition and the type of activity in the room is active action.

Realisation of the action, especially semi-active and active action, is dependent on cognitive computing and automatically controlled actuating devices. The recently rapid development of computing intelligence has already laid a solid foundation. As for automatically controlled actuating devices, there are motorised, built-in controllers, robotic actuators available. With the maturity of the technology, they can be accessed with reasonable prices.

\section{Proposed architecture of Cognitive FM}

A proposed system architecture of Cognitive FM is constructed as displayed in Figure 2. From a bottom-up view, Cognitive FM consists of eight major layers, i.e., the object layer, sensing layer, data layer, and communication layer for perception; computation layer, application layer, actuation layer and evaluation layer for action. Data flow from the lower layers to the upper ones, finally flow back from actuation layer to object layer, forming a perception-action loop, and from evaluation layer to computation layer and application layer, forming an adaptive-learning cycle.

Figure 2 The system architecture of Cognitive FM

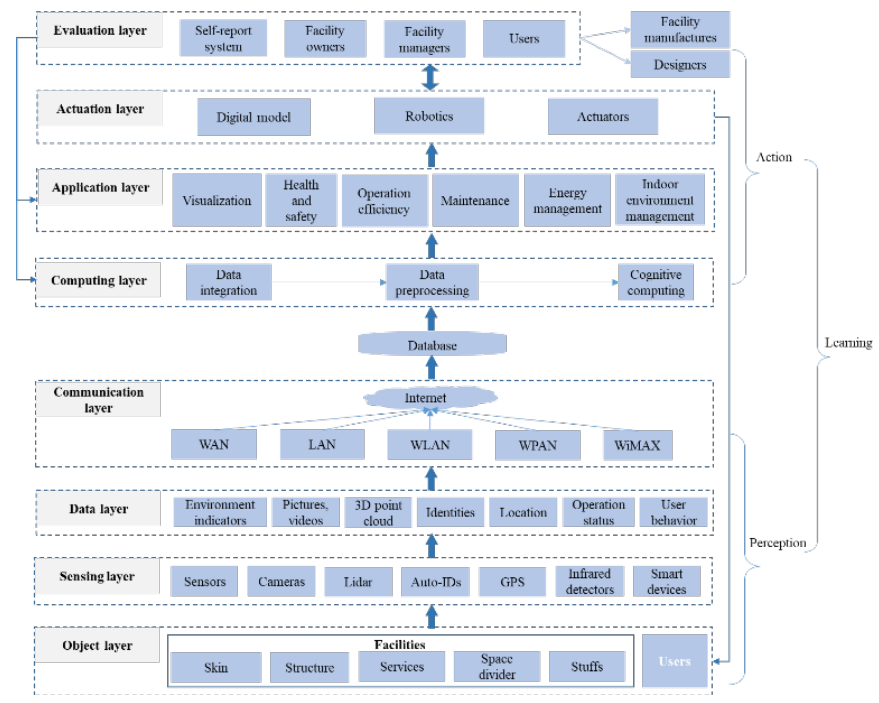

1) The object layer consists of different facilities from physical space and users from social space. The facilities are divided to five 'S's according to Brand (1994), i.e., skin (roofing, siding, exterior door, etc.), structure (beam, column, floors, etc.), services (HVAC, lighting, plumbing, electric power, fire alarm and suppression, phone, etc.), space divider (non-structure wall, interior door, etc.), and stuff (furniture, table, etc.). It is the layer where all commercial, residential, educational or entertaining activities take place, and also the layer people can perceive.
2) The sensing layer, formed by various sensing devices, is to capture features of the object layer. Sensing devices, e.g., sensors, cameras, LiDAR, Auto-IDs, and GPS, sense the environment by processing the incoming stimuli and feedbacks observations to the upper layer. Different sensing devices can sense the status or characteristics of different objects. For example, the occupancy or usage status of a piece of furniture can be collected by sensors; its 3D digital model can be reconstructed by photos or $3 \mathrm{D}$ point cloud data; its identification and location can be accessed by Auto-IDs. Sensors, cameras, Auto-IDs, GPS, IR detectors and most conveniently smartphones are all practicable to record or even analyse users' behaviour.

3) The data layer denotes various data types include environment indicators (temperature, humidity, luminosity, air pressure, density of $\mathrm{CO} 2, \mathrm{CO}, \mathrm{PM} 2.5$ etc.), pictures or videos of a facility or an area, or human's behaviours, 3D point cloud data of a facility, identities of components, furniture and users, location, operation status, user behaviour, and other characteristics.

4) The communication layer helps to upload the sensed data through the Internet to the database. Different types of communication protocols might be used in different circumstances. Local and wireless communication protocols such as WiFi, NFC, and Bluetooth are most promising in FM (Tolman et al., 2009). Emerging personal networks (e.g., WPAN) and new interoperable networks (e.g., WiMAX) will be popular with the emphasising on social aspects.

5) The computation layer expertly analyses the sensed data to generate decision support information through data integration and cognitive computation. Data integration is of great significance because it interoperates heterogeneous data from different sources with different formats. Cognitive computing can embody behaviours such as reasoning, interpreting, inferencing, and learning in an uncertain, fuzzy and complex environment.

6) The application layer uses the information and knowledge abstracted from the lower layer to enable multiple or even massive interactive agents (facilities or users) to reason, plan, and select the most suitable action to support corresponding FM applications. In Cognitive FM, applications of all kinds, including digital visualisation of the facility, health and safety management of both users and facilities, space utilisation optimisation, maintenance planning and arrangement, energy management and indoor environment management, will be supported by facilities augmented with cognition.

7) The actuation layer is where actuators act to facilitate the applications, control the objects and change the perceptron via actuators or robotics. To empower in-time actuating, a detailed digital model of the facility is desired. The model can also be used to provide a user interface, be it a webpage, an APP, or a plug-in, where human and the machine can interact. Through the interface, users or facility managers can give an order to actuators. The actuators will directly control the facilities in the object layer, release alerts or guidance to facility users or 
managers. Robots with caring, operation or maintenance abilities can also conduct actuations.

8) The evaluation layer shares import interfaces with social networks, on which facility managers and users can share ondemand service provisioning. Novel performance metrics are designed to evaluate the provisioned services and feedback the evaluation result to the cognition process. Cognitive FM also has a self-report system to update the performance data every day/month/quarter/year, and even every hour. Moreover, the evaluation results will feedback to the application layer to reset the application variables and objectives, or to the computation layer to adjust the computing algorithms, enabling its adaptivelearning. With adaptive-learning, a cognitive facility will learn from the experience of itself and others, just like the way human beings learn from themselves and others. They can update and adapt their knowledge according to the changing of environment, requirements, and targets. Besides, some relating evaluations can also be sent back to facility designer or manufacture for refined facility design and production.

This eight-layer system architecture of Cognitive FM facilitates its three key elements, i.e., perception, learning, and action. Sensing characteristics of facilities and users as the perception stimuli and action as the output, Cognitive FM energises multi-sourcing and cognitive computing that directly supports various applications. Within Cognitive FM, users, facility managers/owners, and facility manufacturers/designers are included in the loop for data sensing, information analysis, decision making, and application executing, constructing a comprehensive system representing the complex real world. Besides, via the perception-action cycle and adaptive-learning cycle, the system can achieve cognitive control and adaptive learning.

\section{Conclusion}

This paper proposed the concept of cognitive facility management (FM) which aims to add advanced active intelligence that mimics the mechanism of human brain information processing to FM. The definition of Cognitive FM was given as the active intelligent management of a facility, which can perceive through cognitive systems, learn in the manner of human cognition with the power of cognitive computing, and act actively, adaptively, and efficiently via automated actuators, to improve the quality of people's life and productivity of core business. Along with the definition of Cognitive FM, an architecture, inspired by human cognition system, to add cognition to FM revealed. With the eight layers, i.e., the object layer, sensing layer, data layer, and communication layer for perception, computation layer, application layer, actuation layer and evaluation layer for action, active intelligence of FM found its way to stand out. The essence of the architecture is two cycles, i.e., the perception-action and adaptive-learning cycle. The former one accomplishes the control of the facilities according to its perception of the internal and external environment; while the latter gives room for learning, adaptation, and evolution, the way how cognition developed. This architecture puts most of the advanced technologies of information, communication, computation, and action together.

Different from passive, pre-setting and nonreciprocal intelligence of smart building and intelligent building, Cognitive FM proposes an active, adaptive and naturally interactive way to give intelligence to the facility from the perspectives of the facility manager. The facility can understand its users, interact and serve for them as an experienced but agile manager in both perception and action. It is the best way we can imagine that facilities and human beings can interact, cooperate, and symbioses, although it may take a long way for this novel and pioneering concept to land in practice. However, with the systematic architecture, pervasive supporting technologies that are already there, and more importantly, the endeavours of both academia and industry, it should not be long before its realisation. Real life cases will be conducted with collaboration from the industry. This paper acts as the first stone of the endeavour and inspires more efforts to add cognition to FM.

\section{References}

Alexander K (2013) Facilities management: theory and practice. Routledge, London, UK.

Atkin B and Brooks A (2015) Total Facility Management (Fourth edition). John Wiley \& Sons, New York, USA.

Bilgen S (2014) Structure and environmental impact of global energy consumption. Renewable and Sustainable Energy Reviews, 38, 890-902.

Brand S (1994) How buildings learn: What happens after they're built. Penguin, New York, USA.

Cavka HB et al. (2017). Developing owner information requirements for BIM-enabled project delivery and asset management. Automation in Construction, 83, 169-183.

Cheng MY et al. (2017). BIM integrated smart monitoring technique for building fire prevention and disaster relief. Automation in Construction, 84, 14-30.

Chotipanich S (2004). Positioning facility
management. Facilities, 22(13/14), 364-372.

Coccoli M et al. (2016). The role of big data and cognitive computing in the learning process. Journal of Visual Languages \& Computing, 38, 97-103.

Ding G et al. (2018). An amateur drone surveillance system based on the cognitive Internet of Things. IEEE Communications Magazine, 56(1), 29-35.

Feng S et al. (2017). Smart home: Cognitive interactive people-centric Internet of Things. IEEE Communications Magazine, 55(2), 34-39.

Franchak JM et al. (2010). Learning by doing: Action performance facilitates affordance perception. Vision research, 50(24), 2758-2765.

Franklin S et al. (2014). LIDA: A systems-level architecture for cognition, emotion, and learning. IEEE Transactions on Autonomous Mental Development, 6(1), 19-41.

Fuster JM (2003). Cortex and mind: Unifying cognition. Oxford university press, New York, USA. 
Ghahramani A et al. (2014). A knowledge based approach for selecting energy-aware and comfort-driven HVAC temperature set points. Energy and Buildings, 85, 536-548.

Gibson JJ (2015). The ecological approach to visual perception: classic edition. Psychology Press, New York, USA.

Giffinger R et al. (2007). City-ranking of European mediumsized cities. Center of Regional Science, Vienna University of Technology, Vienna, Austria, pp. 1-12.

Haykin S (2012). Cognitive dynamic systems: perceptionaction cycle, radar and radio. Cambridge University Press, Cambridge, UK.

Hecht H et al. (2001). Motor learning enhances perceptual judgment: A case for action-perception transfer. Psychological research, 65(1), 3-14.

$\mathrm{Hu} \mathrm{ZZ}$ et al. (2018). BIM-based integrated delivery technologies for intelligent MEP management in the operation and maintenance phase. Advances in Engineering Software, 115, 1-16.

Huang Q and Mao C (2017). Occupancy estimation in smart building using hybrid $\mathrm{CO} 2 /$ light wireless sensor network. Journal of Applied Sciences and Arts, 1(2), 5.

Immordino-Yang MH (2008). The smoke around mirror neurons: Goals as sociocultural and emotional organizers of perception and action in learning. Mind, Brain, and Education, 2(2), 67-73.

IFMA (2017). What is Facilities management? https://www.ifma.org/about/what-is-facility-management (accessed 12/11/2018).

Isikdag U et al. (2013). A BIM-Oriented Model for supporting indoor navigation requirements. Computers, Environment and Urban Systems, 41, 112-123.

ISO (2017). Facility management - Vocabulary (ISO 41011: 2017). $\quad$ https://www.iso.org/obp/ui/\#iso:std:iso:41011:ed1:v1:en (accessed 12/11/2018).

Jazizadeh F et al. (2014). User-led decentralized thermal comfort driven HVAC operations for improved efficiency in office buildings. Energy and Buildings, 70, 398-410.

Kaplan RS and Norton DP (2004). Strategy Maps: Converting Intangible Assets into Tangible Outcomes. Harvard Business Publishing, Massachusetts, USA.

Kim J et al. (2018). Personal comfort models-A new paradigm in thermal comfort for occupant-centric environmental control. Building and Environment, 132, 114-124.

Kotseruba I and Tsotsos JK (2018). 40 years of cognitive architectures: core cognitive abilities and practical applications. Artificial Intelligence Review, 1-78.

Lau D et al. (2013). A cloud-based approach for smart facilities management. In Prognostics and Health Management (PHM), 2013 IEEE Conference. IEEE. pp. 1-8

Leite F and Akinci B (2011). Formalized representation for supporting automated identification of critical assets in facilities during emergencies triggered by failures in building systems. Journal of Computing in Civil Engineering, 26(4), 519-529.
Li $\mathrm{N}$ et al. (2014). A BIM centered indoor localization algorithm to support building fire emergency response operations. Automation in Construction, 42, 78-89.

Lu Q et al. (2018). Activity theory-based analysis of BIM implementation in building O\&M and first response. Automation in Construction, 85, 317-332.

Marchevsky AM et al. (2017). Evidence-based pathology in its second decade: toward probabilistic cognitive computing. Human pathology, 61, 1-8.

Nielsen SB et al. (2016). Sustainability in facilities management: an overview of current research. Facilities, 34(9/10), 535-563.

Niu Y et al. (2016). Smart construction objects. Journal of Computing in Civil Engineering, 30(4), 04015070.

Noda K et al. (2014). Multimodal integration learning of robot behavior using deep neural networks. Robotics and Autonomous Systems, 62(6), 721-736.

Norman DA (1980). Twelve issues for cognitive science. Cognitive science, 4(1), 1-32.

Parn EA et al. (2017). The building information modelling trajectory in facilities management: A review. Automation in Construction, 75, 45-55.

Preissl R et al. (2012). Compass: A scalable simulator for an architecture for cognitive computing. In Proceedings of the International Conference on High Performance Computing, Networking, Storage and Analysis. IEEE Computer Society Press, pp. 1-11.

Ratey JJ (2001). A user's guide to the brain: Perception, attention, and the four theatres of the brain. Vintage Books, New York, USA.

Rondeau EP et al. (2012). Facility management. John Wiley \& Sons, New York, USA.

Rookes P and Willson J (2005). Perception: Theory, Development and Organisation. Routledge, London, UK.

Russell SJ and Norvig P (2016). Artificial intelligence: $a$ modern approach. Pearson Education, New Jersey, USA.

Sheth A (2016). Internet of things to smart IoT through semantic, cognitive, and perceptual computing. IEEE Intelligent Systems, 31(2), 108-112.

Somov A et al. (2013). Supporting smart-city mobility with cognitive Internet of Things. In Future Network and Mobile Summit, IEEE, pp. 1-10.

Tang $\mathrm{P}$ et al. (2010). Automatic reconstruction of as-built building information models from laser-scanned point clouds: A review of related techniques. Automation in construction, 19(7), 829-843.

Teicholz P (Ed.). (2013). BIM for facility managers. John Wiley \& Sons, New York, USA.

Tolman A et al. (2009). The benefits and obstacles of mobile technology in FM service procurement. Facilities, 27(11/12), 445-456.

Volk R et al. (2014). Building Information Modeling (BIM) for existing buildings-Literature review and future needs. Automation in construction, 38, 109-127. 
Wang Z et al. (2018). Individual difference in thermal comfort: A literature review. Building and Environment, 138, 181-193.

Wong JKW et al. (2018). Digitisation in facilities management: A literature review and future research directions. Automation in Construction, 92, 312-326.

Woods DD and Hollnagel E (2005). Joint cognitive systems: Foundations of cognitive systems engineering. CRC Press, Florida, USA.

Wu Q et al. (2014). Cognitive internet of things: a new paradigm beyond connection. IEEE Internet of Things Journal, 1(2), 129-143.

$\mathrm{Xu} \mathrm{J}$ et al. (2018a). 3D point cloud data enabled facility management: A critical review. In The 23rd International Symposium on the Advancement of Construction Management and Real Estate, Guiyang, China.

$\mathrm{Xu} \mathrm{Z}$ et al. (2017). PMV-based event-triggered mechanism for building energy management under uncertainties. Energy and Buildings, 152, 73-85.

Xue $\mathrm{P}$ et al. (2014). The effects of daylighting and human behavior on luminous comfort in residential buildings: A questionnaire survey. Building and Environment, 81, 51-59.

Yang Z and Becerik-Gerber B (2014). The coupled effects of personalized occupancy profile based HVAC schedules and room reassignment on building energy use. Energy and Buildings, 78, 113-122.

Zhang M et al. (2012). Cognitive internet of things: concepts and application example. International Journal of Computer Science Issues, 9(6), 151.

Capuano N and Toti D (2018). Experimentation of a smart learning system for law based on knowledge discovery and cognitive computing. Computers in Human Behavior. (In press). https://doi.org/10.1016/j.chb.2018.03.034.

Dessi D et al. (2018). Bridging learning analytics and Cognitive Computing for Big Data classification in microlearning video collections. Computers in Human Behavior.

(In press). https://doi.org/10.1016/j.chb. 